\title{
Nikolskii inequality and functional classes on compact Lie groups
}

\author{
E. D. Nursultanov, M. V. Ruzhansky, S. Yu. Tikhonov
}

\begin{abstract}
In this note we study Besov, Triebel-Lizorkin, Wiener, and Beurling function spaces on compact Lie groups. A major role in the analysis is played by the Nikolskii inequality.
\end{abstract}

1. Introduction. The classical Nikolskii inequality for trigonometric polynomials $T_{L}$ of degree up to $L$ can be written as ( $\mathbf{N i k 5 1}$ ):

$$
\left\|T_{L}\right\|_{L^{q}(\mathbb{T})} \leq 2 L^{1 / p-1 / q}\left\|T_{L}\right\|_{L^{p}(\mathbb{T})},
$$

where $1 \leq p<q \leq \infty$. The Nikolskii inequality plays an important role in the analysis of different function spaces (for example, see [Tri83]) and in the approximation theory (for example, see [DT05]).

In the Euclidean case, for functions $f \in L^{p}\left(\mathbb{R}^{n}\right)$ such that $\operatorname{supp}(\widehat{f})$ is compact (cf. NW78] we have

$$
\|f\|_{L^{q}\left(\mathbb{R}^{n}\right)} \leq(C(p) \mu(\operatorname{conv}[\operatorname{supp}(\widehat{f})]))^{1 / p-1 / q}\|f\|_{L^{p}\left(\mathbb{R}^{n}\right)},
$$

where $1 \leq p \leq q \leq \infty, \mu(E)$ is the Lebesgue measure of $E$, and $\operatorname{conv}[E]$ is the convex hull of $E$. Inequalities of the form (11) are often called Plancherel-Polya-Nikolskii inequalities.

Recently, in Pes09] Pesenson obtained the Bernstein-Nikolskii inequality on symmetric spaces of noncompact type, and in Pes08] on compact homogeneous spaces.

Let $G$ be a compact Lie group of $\operatorname{dimension} \operatorname{dim} G$ and let $\widehat{G}$ be its unitary dual. If we fix bases in representation spaces we can work with matrix representations $\xi: G \rightarrow \mathbb{C}^{d_{\xi} \times d_{\xi}}$ of dimensions $d_{\xi}$. By the Peter-Weyl theorem the system $\left\{\sqrt{d_{\xi}} \xi_{i j}:[\xi] \in \widehat{G}, 1 \leq i, j \leq d_{\xi}\right\}$ is an orthonormal basis in $L^{2}(G)$ with respect to the normalized Haar measure on $G$. All the integrals below and the spaces $L^{p}(G)$ will be always considered with respect to this normalized bi-invariant Haar measure on $G$.

For $f \in C^{\infty}(G)$ we define its Fourier coefficient at $\xi \in[\xi] \in \widehat{G}$ by

$$
\widehat{f}(\xi)=\int_{G} f(x) \xi(x)^{*} \mathrm{~d} x .
$$

Thus, we have $\widehat{f}(\xi) \in \mathbb{C}^{d_{\xi} \times d_{\xi}}$. The Fourier series of a function $f$ takes the form

$$
f(x)=\sum_{[\xi] \in \widehat{G}} d_{\xi} \operatorname{Tr}(\widehat{f}(\xi) \xi(x)) .
$$

E. Nursultanov was supported by grants MOH PK 4080 GF-4, 3311 GF-4. M. Ruzhansky was supported by EPSRC Leadership Fellowship, EPSRC EP/K039407/1 and Leverhulme Grant RPG-2014-02. S. Tikhonov was supported by grants 2014-SGR-289 from AGAUR (Generalitat de Catalunya), MTM 201459174-P, and RFFI-13-01-00043. 
For $[\xi] \in \widehat{G}$ by $\langle\xi\rangle$ we denote the eigenvalue of the operator $\left(I-\mathcal{L}_{G}\right)^{1 / 2}$ corresponding to the representation class $[\xi] \in \widehat{G}$, where $\mathcal{L}_{G}$ is the Laplacian on $G$, see, for example [Ste70, Chapter 1.7].

In RT10 the following Lebesgue spaces $\ell^{p}(\widehat{G})$ on $\widehat{G}$ were defined as follows: using the Fourier coefficients of $f$, we set

$$
\|\widehat{f}\|_{\ell^{p}(\widehat{G})}=\left(\sum_{[\xi] \in \widehat{G}} d_{\xi}^{p\left(\frac{2}{p}-\frac{1}{2}\right)}\|\widehat{f}(\xi)\|_{\mathrm{HS}}^{p}\right)^{1 / p}, 1 \leq p<\infty,
$$

and

$$
\|\widehat{f}\|_{\ell \infty(\widehat{G})}=\sup _{[\xi] \in \widehat{G}} d_{\xi}^{-\frac{1}{2}}\|\widehat{f}(\xi)\|_{\mathrm{HS}},
$$

where $\|\widehat{f}(\xi)\|_{\text {HS }}=\operatorname{Tr}\left(\widehat{f}(\xi) \widehat{f}(\xi)^{*}\right)^{1 / 2}$. For these spaces the following Hausdorff-Young inequalities are valid:

$$
\|\widehat{f}\|_{\ell^{p^{\prime}}(\widehat{G})} \leq\|f\|_{L^{p}(G)},\|f\|_{L^{p^{\prime}(G)}} \leq\|\widehat{f}\|_{\ell^{p}(\widehat{G})}, \quad 1 \leq p \leq 2, \quad \frac{1}{p^{\prime}}+\frac{1}{p}=1 .
$$

Let $N(L)$ be the Weyl eigenvalue counting function for the elliptic pseudo-differential operator $\left(1-\mathcal{L}_{G}\right)^{1 / 2}$, denoting the number of its eigenvalues $\leq L$ counted with multiplicities. Then

$$
N(L)=\sum_{\substack{\langle\xi\rangle \leq L \\[\xi] \in \widehat{G}}} d_{\xi}^{2}
$$

For sufficiently large $L$ the Weyl asymptotic formula says that

$$
N(L) \sim C_{0} L^{n}, \quad C_{0}=(2 \pi)^{-n} \int_{\sigma_{1}(x, \omega)<1} \mathrm{~d} x \mathrm{~d} \omega,
$$

where $n=\operatorname{dim} G$, and the integral is taken with respect to the canonical measure on the cotangent bundle $T^{*}(G)$ induced by the canonical symplectic form. Here $\sigma_{1}$ is the principal symbol of the operator $\left(1-\mathcal{L}_{G}\right)^{1 / 2}$, see e.g. [Shu01].

Full proofs of our results below will appear in [NRT15.

2. Nikolskii inequality. Let $T$ be a trigonometric polynomial on a compact Lie group $G$, i. e. a function with only finitely many non-zero Fourier coefficients. Let $D$ be the Dirichlet kernel, i.e. the function $D \in C^{\infty}(G)$ such that

$$
\widehat{D}(\xi):=I_{d_{\xi}} \quad \text { for }\langle\xi\rangle \leq L,
$$

and zero otherwise. Here $I_{d_{\xi}} \in \mathbb{C}^{d_{\xi} \times d_{\xi}}$ denote the identity matrix.

Theorem 1. Let $0<p<q \leq \infty$. For $0<p \leq 2$ set $\rho:=1$, and for $2<p<\infty$ let $\rho$ be the smallest integer $\geq p / 2$. Then

$$
\|T\|_{L^{q}(G)} \leq\left(\sum_{\widehat{T^{\rho}}(\xi) \neq 0} d_{\xi}^{2}\right)^{\frac{1}{p}-\frac{1}{q}}\|T\|_{L^{p}(G)}
$$

Moreover, this inequality is sharp for $p=2$ and $q=\infty$, and the equality is attained at $T=D$.

We note that for the classical trigonometric polynomials of several variables the Nikolskii inequality is well known ([Nik51]). 
REMARK 2. Note that if $T=T_{L}$, i.e. if $\widehat{T}(\xi)=0$ for $\langle\xi\rangle>L$, then $\sum_{\widehat{T}(\xi) \neq 0} d_{\xi}^{2} \leq N(L)$ and, therefore,

$$
\left\|T_{L}\right\|_{L^{q}(G)} \leq N(\rho L)^{\frac{1}{p}-\frac{1}{q}}\left\|T_{L}\right\|_{L^{p}(G)} \asymp(\rho L)^{n\left(\frac{1}{p}-\frac{1}{q}\right)}\left\|T_{L}\right\|_{L^{p}(G)} .
$$

For a partial sum of the Fourier series of $f$ :

$$
S_{L} f(x)=\sum_{\langle\xi\rangle \leq L} d_{\xi} \operatorname{Tr}(\widehat{f}(\xi) \xi(x))
$$

one can prove the following result.

Corollary 3. Let $G$ be a compact Lie group and let $1 \leq p<q \leq \infty$ be such that $\frac{1}{p}>\frac{1}{q}+\frac{1}{2}$. Then we have

$$
\left(\sum_{k=1}^{\infty} \frac{\left(k^{1-1 / p+1 / q} \sup _{N(L) \geq k} \frac{1}{N(L)}\left\|S_{L} f\right\|_{L^{q}(G)}\right)^{p}}{k}\right)^{1 / p} \leq C\|f\|_{L^{p}(G)}
$$

for all $f \in L^{p}(G)$. In particular, we have $N(L)^{\frac{1}{q}-\frac{1}{p}}\left\|S_{L} f\right\|_{L^{q}(G)}=o(1)$ as $L \rightarrow \infty$.

3. Embeddings of functional classes. Here we investigate embedding theorems and interpolation properties of several classes of functions on a compact Lie group $G$. Using the definition (2) of the Fourier series, we can defined Sobolev, Besov, and Triebel-Lizorkin spaces, respectively, as follows:

$$
\begin{gathered}
H_{p}^{r}=H_{p}^{r}(G)=\left\{f \in \mathcal{D}^{\prime}(G):\|f\|_{H_{p}^{r}}:=\left\|\left(1-\mathcal{L}_{G}\right)^{r / 2} f\right\|_{p}<\infty\right\}, \\
B_{p, q}^{r}=B_{p, q}^{r}(G)=\left\{f \in \mathcal{D}^{\prime}(G):\|f\|_{B_{p, q}^{r}}:=\left(\sum_{s=0}^{\infty} 2^{s r q}\left\|_{2^{s} \leq\langle\xi\rangle<2^{s+1}} d_{\xi} \operatorname{Tr}(\widehat{f}(\xi) \xi(x))\right\|_{p}^{q}\right)^{1 / q}<\infty\right\}, \\
F_{p, q}^{r}=F_{p, q}^{r}(G)=\left\{f \in \mathcal{D}^{\prime}(G):\|f\|_{F_{p, q}^{r}}:=\left\|\left(\sum_{s=0}^{\infty} 2^{s r q}\left|\sum_{2^{s} \leq\langle\xi\rangle<2^{s+1}} d_{\xi} \operatorname{Tr}(\widehat{f}(\xi) \xi(x))\right|^{q}\right)^{1 / q}\right\|_{p}<\infty\right\} .
\end{gathered}
$$

Then we have the following result:

TheOrem 4. Let $G$ be a compact Lie group of dimension $n$. Then

$$
\text { (2) } B_{p, \min \{p, 2\}}^{r} \hookrightarrow H_{p}^{r} \hookrightarrow B_{p, \max \{p, 2\}}^{r}, \quad r \in \mathbb{R}, \quad 1<p<\infty \text {; }
$$$$
0<p_{1} \leq p_{2} \leq \infty, 0<q \leq \infty, r_{2}=r_{1}-n\left(\frac{1}{p_{1}}-\frac{1}{p_{2}}\right)
$$

(3) $\quad B_{p, q}^{r} \hookrightarrow L_{q}, \quad 1<p<q<\infty, r=n\left(\frac{1}{p}-\frac{1}{q}\right)$;

(4) $B_{p, 1}^{r} \hookrightarrow L_{\infty}, \quad 0<p \leq \infty, \quad r=\frac{n}{p}$;

(5) $\quad B_{p, \min \{p, q\}}^{r} \hookrightarrow F_{p, q}^{r} \hookrightarrow B_{p, \max \{p, q\}}^{r}, \quad 1<p<\infty, 0<p<\infty, 0<q \leq \infty$.

(6) $\quad\left(B_{p, \beta_{0}}^{r_{0}}, B_{p, \beta_{1}}^{r_{1}}\right)_{\theta, q}=\left(H_{p}^{r_{0}}, H_{p}^{r_{1}}\right)_{\theta, q}=\left(F_{p, \beta_{0}}^{r_{0}}, F_{p, \beta_{1}}^{r_{1}}\right)_{\theta, q}=B_{p, q}^{r}, \quad 0<r_{1}<r_{0}<\infty$,

$$
0<\beta_{0}, \beta_{1}, q \leq \infty, 1<p<\infty, r=(1-\theta) r_{0}+\theta r_{1}, \quad 0<\theta<1 .
$$

For functions on the torus the corresponding results can be found, for example, in the book Tri83.

Consequently, using norms (3) and (44), we can investigate the embeddings between Wiener and Beurling classes defined as follows:

$$
A^{\beta}(\widehat{G})=\left\{f \in \mathcal{D}^{\prime}(G):\|f\|_{A^{\beta}}:=\|\widehat{f}\|_{\ell^{\beta}(\widehat{G})}=\left(\sum_{[\xi] \in \widehat{G}} d_{\xi}^{\beta\left(\frac{2}{\beta}-\frac{1}{2}\right)}\|\widehat{f}(\xi)\|_{\mathrm{HS}}^{\beta}\right)^{1 / \beta}<\infty\right\}
$$


and

$$
A^{*, \beta}(\widehat{G})=\left\{f:\|f\|_{A^{*, \beta}(\widehat{G})}:=\left(\sum_{s=0}^{\infty} 2^{n s}\left(\sup _{2^{s} \leq\langle\xi\rangle} d_{\xi}^{-1 / 2}\|\widehat{f}(\xi)\|_{\mathrm{HS}}\right)^{\beta}\right)^{1 / \beta}<\infty\right\},
$$

where $0<\beta<\infty$. For periodic functions, i.e. for $G=\mathbb{T}^{n}$, we have $d_{\xi} \equiv 1, \widehat{G} \simeq \mathbb{Z}^{n}$, and $\|\widehat{f}(\xi)\|_{\text {HS }}=|\widehat{f}(\xi)|$, and such spaces have been investigated, for example, in Beu48, BLT97] and [TB04, Ch. 6].

THEOREM 5. Let $G$ be a compact Lie group of dimension $n$.

(A). Let $\alpha>0$ and $\frac{1}{\beta}=\frac{n}{\alpha}+\frac{1}{p^{\prime}}$. Then

$$
\begin{aligned}
& \|f\|_{A^{\beta}} \leq C\|f\|_{B_{p, \beta}^{\alpha}}, \quad 1<p \leq 2 ; \\
& \|f\|_{B_{p, \beta}^{\alpha}} \leq C\|f\|_{A^{\beta}}, \quad 2 \leq p<\infty .
\end{aligned}
$$

(B). Let $0<\beta<\infty \quad p \geq 2$. Then

$$
C_{1}\|f\|_{B_{p, \beta}^{n\left(\frac{1}{\beta}-\frac{1}{p^{\prime}}\right)}} \leq\|f\|_{A^{*, \beta}} \leq C_{2}\|f\|_{B_{1, \beta}^{n / \beta}}
$$

As a consequence, we obtain

$$
C_{1}\|f\|_{A^{\beta}} \leq\|f\|_{B_{2, \beta}^{n(1 / \beta-1 / 2)}} \leq C_{2}\|f\|_{A^{*, \beta}} .
$$

The left inequality is an analogue of Bernstein theorem on the absolute convergence of Fourier series. It strengthens the following inequality proved by Faraut in Far08 for groups $G$ of unitary matrices: if $f \in C^{k}(G)$ for an even $k>\frac{\operatorname{dim} G}{2}$ then $\widehat{f} \in \ell^{1}(\widehat{G})$, i.e. $f \in A(G)$. For periodic functions of several variables inequality (5) follows from the results in MS47.

Finally, we look at the following Beurling-type spaces:

$$
A_{r}^{*, \beta}=\left\{f:\|f\|_{A_{r}^{*, \beta}}:=\left(\sum_{s=0}^{\infty}\left(2^{r n s} \sup _{2^{s} \leq\langle\xi\rangle} d_{\xi}^{-1 / 2}\|\widehat{f}(\xi)\|_{\mathrm{HS}}\right)^{\beta}\right)^{\frac{1}{\beta}}<\infty\right\} .
$$

Note that $A_{1 / \beta}^{*, \beta}=A^{*, \beta}$. These spaces are interpolation spaces in the following sense: Then

Theorem 6. Let $0<r_{1}<r_{0}<\infty, 0<\beta_{0}, \beta_{1}, q \leq \infty, r=(1-\theta) r_{0}+\theta r_{1} \quad 0<\theta<1$.

In particular,

$$
\left(A_{r_{0}}^{*, \beta_{0}}, A_{r_{1}}^{*, \beta_{1}}\right)_{\theta, q}=A_{r}^{*, q} .
$$

$$
\left(A^{*, 1 / r_{0}}, A^{*, 1 / r_{1}}\right)_{\theta, 1 / r}=A^{*, 1 / r}
$$

\section{References}

[BLT97] E. S. Belinskii, E. R. Lifyand, and R. M. Trigub, The Banach algebra $A^{*}$ and its properties, J. Fourier Anal. Appl., 3(2):103-129, 1997.

[Beu48] A. Beurling, On the spectral synthesis of bounded functions, Acta Math., 81:14, 225-238, 1949.

[DT05] Z. Ditzian and S. Tikhonov, Ul'yanov and Nikol'skii-type inequalities. J. Approx. Theory, 133(1), 100-133, 2005.

[Far08] J. Faraut, Analysis on Lie groups, Cambridge, 2008.

[MS47] S. Minakshisundaram and O. Szász, On absolute convergence of multiple Fourier series, Trans. Amer. Math. Soc. 61, 36-53, 1947.

[NW78] R. J. Nessel and G. Wilmes, Nikolskii-type inequalities for trigonometric polynomials and entire functions of exponential type. J. Austral. Math. Soc. Ser. A, 25(1), 7-18, 1978. 
[Nik51] S. M. Nikolsky, Inequalities for entire functions of finite degree and their applications in the theory of differentiable functions of many variables. Proc. Steklov Math. Inst., 38, 244-278, 1951.

[NRT15] E. Nursultanov, M. Ruzhansky and S. Tikhonov, Nikolskii inequality and Besov, Triebel-Lizorkin, Wiener and Beurling spaces on compact homogeneous manifolds, Ann. Sc. Norm. Super. Pisa Cl. Sci., to appear. arXiv:1403.3430

[Pes08] I. Pesenson, Bernstein-Nikolskii inequalities and Riesz interpolation formula on compact homogeneous manifolds. J. Approx. Theory, 150(2), 175-198, 2008.

[Pes09] I. Pesenson, Bernstein-Nikolskii and Plancherel-Polya inequalities in Lp-norms on non-compact symmetric spaces. Math. Nachr., 282(2), 253-269, 2009.

[RT10] M. Ruzhansky and V. Turunen, Pseudo-differential operators and symmetries. Background analysis and advanced topics, volume 2 of Pseudo-Differential Operators. Theory and Applications. Birkhäuser Verlag, Basel, 2010.

[Shu01] M. A. Shubin, Pseudodifferential operators and spectral theory. Springer-Verlag, Berlin, second edition, 2001. Translated from the 1978 Russian original.

[Ste70] E. Stein, Topics in harmonic analysis related to the Littlewood-Paley theory, Princeton Uversity Press, 1970.

[Tri83] H. Triebel, Theory of function spaces, Birkhäuser, Verlag, Basel, 1983.

[TB04] R. M. Trigub and E. S. Bellinsky, Fourier analysis and approximation of functions, Kluwer Academic Publishers, Dordrecht, 2004.

E. D. Nursultanov:

Moscow State University (Kazakhstan Branch)

AND Eurasian National University

ASTANA

KAZAKHSTAN

E-mail address er-nurs@yandex.ru

M. V. RuZHANSKY:

Department of Mathematics

IMPERIAL COLLEGE LONDON

180 QueEn's Gate, London SW7 2AZ

UNITED KINGDOM

E-mail address m.ruzhansky@imperial.ac.uk

S. Yu. Tikhonov:

ICREA And Centre de Recerca Matemàtica (CRM)

E-08193, Bellaterra

BARCELONA

E-mail address stikhonov@crm.cat 